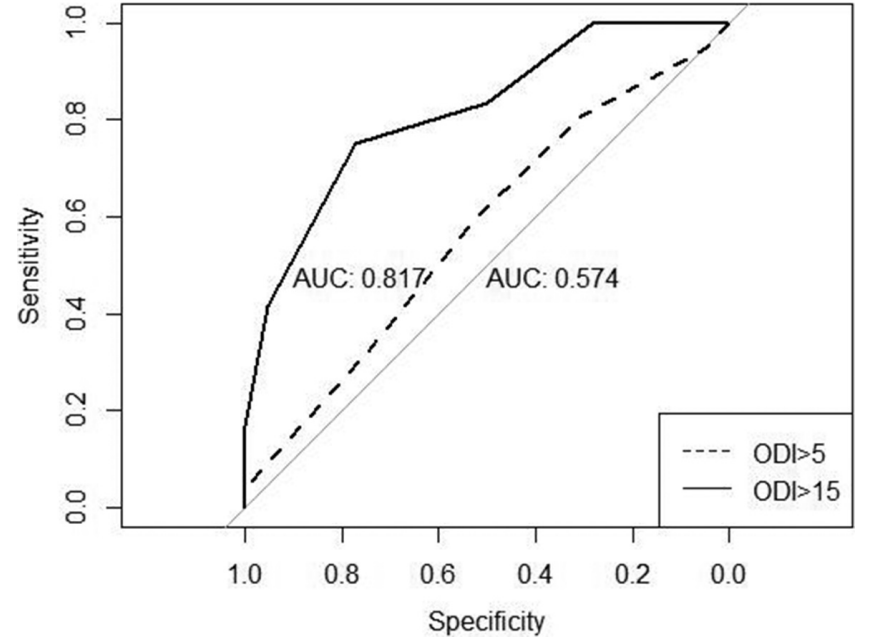

Abstract S27 Figure 1 ROC curves for STOPBANG to predict ODI $\geq 5$ and $\mathrm{ODI} \geq 15$

Conclusion Predictive performance of STOPBANG in our patient cohort at the conventional cut off value was poor. The probable explanation is that the cardiac surgical population is preselected as male, older and most suffer with hypertension. Thus the majority will score as high risk for OSA. STOPBANG had no prognostic value on worse postoperative outcomes in our study, which again contrasts with the findings in general surgical cohorts.

\section{S28 EFFECT OF SLEEP APNOEA ON POST-OPERATIVE OUTCOMES IN CARDIAC SURGERY}

M Mason, J Hernández-Sánchez, D Horton, A Clutterbuck-James, I Smith. Papworth Hospital NHS Foundation Trust, Cambridge, UK

\subsection{6/thoraxjnl-2015-207770.34}

Introduction and objectives Obstructive sleep apnoea (OSA) is common and can be associated with adverse health outcomes. There are conflicting data for the impact of undiagnosed OSA on the outcome of surgical procedures but at least some results suggest an association with worse outcomes. EuroSCORE risk model was developed to calculate the risk of mortality after cardiac surgery. We evaluated the prevalence and impact of undiagnosed sleep apnoea (SA) on postoperative outcomes in cardiac surgery.

Methods Patients undergoing coronary artery bypass grafting with or without cardiac valve surgery were screened for the presence of SA, prior to surgery, with the STOPBANG questionnaire and overnight oximetry. SA was defined as a 4\% oxygen desaturation index (ODI) of $\geq 5 / \mathrm{hr}$. A Weibull model was used to analyse lengths of stay (LoS) in intensive care unit (ICU). Complications in ICU were dichotomised and analysed with binary logistic regressions. Parsimonious models were obtained using a combination of step-wise regression and manually removing predictors that did not reach the 5\% significance level.

Results 122 subjects were included in final analysis of which 57 (47\%) had a new diagnosis of SA. Of those, 45 (79\%) had mild SA and $12(21 \%)$ had moderate/severe SA. There was no simple relationship between OSA as measured by ODI and LoS in ICU. The most significant predictor for ICU LoS was developing complications at ICU $(\mathrm{p}<0.001)$. The independent predictors associated with increasing likelihood of developing major organ complications following cardiac surgery were EuroSCORE, ODI and intravenous opioid analgesia (IOA). When patients with mild and moderate SA received IOA, predicted probability of complications rose 2.4 and 1.4 times respectively (Figure 1).

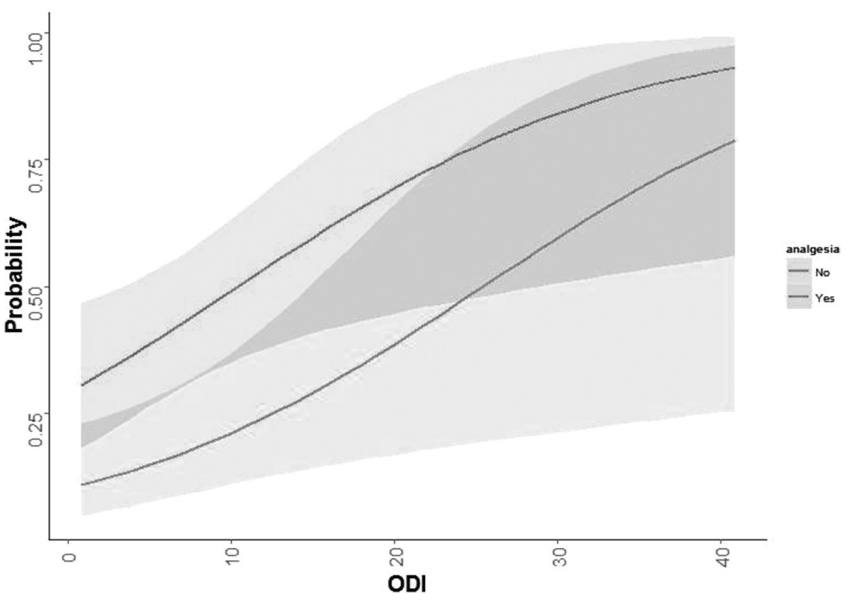

Abstract S28 Figure 1 Predicted probabilities and $95 \% \mathrm{Cl}$ of suffering a complication at ICU as ODI increases for individuals with average EuroSCORE (5) and with or without IOA

Conclusion We found a high prevalence of undiagnosed sleep apnoea in our cohort. EuroSCORE, SA and the administration of intravenous morphine were found to be independent risk factors for developing post-operative complications. This risk has increased when patients with SA received intravenous morphine.

\section{S29 PREDICTORS OF CONTINUOUS POSITIVE AIRWAYS PRESSURE USAGE AT SIX MONTHS IN MINIMALLY SYMPTOMATIC PATIENTS. FURTHER DATA FROM THE MOSAIC TRIAL}

${ }^{1} \mathrm{CD}$ Turnbull, ${ }^{2} \mathrm{DJ}$ Bratton, ${ }^{3} \mathrm{SE}$ Craig, ${ }^{2} \mathrm{M}$ Kohler, ${ }^{1} \mathrm{JR}$ Stradling. ${ }^{1}$ Oxford Centre for Respiratory Medicine and NIHR Oxford Biomedical Research Centre, Oxford, UK: ${ }^{2}$ University Hospital Zurich, Zurich, Switzerland; ${ }^{3}$ Aintree Chest Centre, Liverpool, UK

\subsection{6/thoraxjnl-2015-207770.35}

Introduction Severity of OSA and early patterns of CPAP usage have previously been shown to determine subsequent long term CPAP use in patients with symptomatic moderate-to-severe disease. ${ }^{1}$ We wished to see if different factors influenced compliance in minimally symptomatic patients.

Methods Patients were randomised to 6-months of CPAP or standard care if they had an ODI of $>7.5 \mathrm{~h}$ due to OSA on a baseline sleep study, but had insufficient daytime OSA symptoms to mandate $\mathrm{CPAP}^{2}$

Baseline characteristics (Table 1), medical history, ESS, SAQLI and SF-36 were recorded. Repeat overnight pulse oximetry was performed after entry for uniformity of trial ODI across recruiting centres. 
CPAP usage data were downloaded at the 2-4 week assessment, and at the 6 month assessment. Those who withdrew were assumed to have 0:00 $\mathrm{h} / \mathrm{n}$ usage.

Correlations were calculated between CPAP usage at the 6 month assessment and both the baseline characteristics and to the 2-4 week CPAP usage data.

\begin{tabular}{|c|c|c|}
\hline Variable & $\begin{array}{l}\text { Mean (SD), Median (IQR) or } \\
\text { Number (\%) }\end{array}$ & $\begin{array}{l}100 \% \\
\text { Range }\end{array}$ \\
\hline Age (mean; SD) & $57.9(7.2)$ & $45-75$ \\
\hline Gender (number male;\%) & $153(78.5 \%)$ & - \\
\hline Ethnicity (number white;\%) & $188(96.4 \%)$ & - \\
\hline BMI (mean kg/m²; SD) & $32.2(5.6)$ & $21.6-51.6$ \\
\hline \multicolumn{3}{|l|}{ Smoking status (number;\%) } \\
\hline Current & $17(8.7 \%)$ & - \\
\hline Ex-smoker & $102(52.3 \%)$ & - \\
\hline Never smoker & $76(39 \%)$ & - \\
\hline Reported snoring (number yes;\%) & $190(97.4 \%)$ & - \\
\hline Reported apnoeas (number yes;\%) & $142(72.8 \%)$ & - \\
\hline Reported choking (number yes;\%) & $66(33.8 \%)$ & - \\
\hline Reported nocturia (number yes;\%) & $114(58.5 \%)$ & - \\
\hline $\begin{array}{l}\text { Oxygen desaturation index (ODI, } \\
\text { median; IQR) }\end{array}$ & $10.2(4.7,17.5)$ & $0.5-58.0$ \\
\hline Epworth Sleepiness Score (ESS, mean; SD) & $7.9(4.4)$ & -18 \\
\hline $\begin{array}{l}\text { Short sleep apnoea quality of life index } \\
\text { (SAQLI, mean; SD) }\end{array}$ & $4.9(1.1)$ & $2.3-6.9$ \\
\hline SF-36 Physical Summary (mean; SD) & $42.0(12.3)$ & $9.4-61.3$ \\
\hline SF- 36 Mental Summary (mean; SD) & $48.1(10.3)$ & 19.9-63.7 \\
\hline
\end{tabular}

Results Median CPAP usage at 2-4 week follow-up was low at $2: 49 \mathrm{~h} / \mathrm{n}(\mathrm{n}=174$, IQR $0: 44,5: 13)$. Median usage at 6 month follow-up was $2: 17 \mathrm{~h} / \mathrm{n}(\mathrm{n}=195$, IQR 0:08, 4:54).

At 6 months males had significantly greater mean usage at $2: 56 \mathrm{~h} / \mathrm{n}$ compared to $1: 47 \mathrm{~h} / \mathrm{n}$ in females $(95 \%$ confidence intervals of the difference, $-1: 49$ to $-0: 09 \mathrm{~h} / \mathrm{n}, \mathrm{p}=0.02)$. There were no other significant predictors of 6 month usage (age, BMI, ODI, ESS, sleep symptoms, smoking status, ethnicity, SAQLI, SF-36).

Average usage of CPAP at 2-4 week assessment was moderately correlated with the average usage at the 6 month assessment $(\mathrm{r}=0.76, \mathrm{p}<0.001)$.

Conclusions Male gender predicted greater CPAP usage at 6 months, but no other baseline characteristics were predictive of CPAP usage in these minimally symptomatic patients with generally mild OSA. 2-4 week CPAP usage was predictive of 6 month usage, but by no means could all patients' usage be predicted at such an early stage. Thus in clinical practice, trials of CPAP are necessary in patients with minimally symptomatic OSA but it may be necessary for patients to try CPAP for longer than one month to determine those benefitting from treatment in the long term.

\section{S30 NUTRITION AND EXERCISE REHABILITATION IN OBESITY HYPOVENTILATION SYNDROME (NERO): A PILOT RANDOMISED CONTROLLED TRIAL}

${ }^{1} \mathrm{~S}$ Mandal, 'ES Suh, ${ }^{1} \mathrm{R}$ Harding, ${ }^{1} \mathrm{~A}$ Vaughan-France, ${ }^{1} \mathrm{M}$ Ramsay, ${ }^{1} \mathrm{~B}$ Connolly, ${ }^{1} \mathrm{D}$ Bear, ${ }^{2} \mathrm{H}$ McLaughlin, ${ }^{2} \mathrm{~S}$ Greenwood, ${ }^{3} \mathrm{M}$ Polkey, ${ }^{4} \mathrm{M}$ Elliott, ${ }^{5} \mathrm{~A}$ Douiri, ${ }^{2} \mathrm{~J}$ Moxham, ${ }^{1} \mathrm{~N}$ Hart. 'Lane-Fox Respiratory Unit, Guy's and St Thomas' NHS Trust, London, UK; ${ }^{2}$ King's College Hospital, London, UK; ${ }^{3}$ Royal Brompton and Harefield NHS Trust, London, UK; ${ }^{4}$ St James' Hospital, Leeds, Leeds, UK; ${ }^{5}$ Department of Primary Care and Public Health Sciences \& NIHR Biomedical Research Centre at Guy's and St Thomas' NHS Foundation Trust and King's College London, London, UK

\subsection{6/thoraxjnl-2015-207770.36}

Introduction We have previously shown that treatment of obesity hypoventilation syndrome (OHS) with non-invasive ventilation (NIV) results in weight reduction and an increase in physical activity (Murphy et al., 2012). We therefore hypothesised that a multi-modal rehabilitation programme, in addition to NIV, would lead to enhanced weight loss.

Method We conducted a randomised controlled trial of NIV alone vs. NIV and a personalised rehabilitation programme in patients with OHS. Subjects in the intervention group received a bespoke diet and exercise regime, from a dietician and physiotherapist. All patients, in both groups, were reviewed monthly for 3 months. Anthropometrics, exercise capacity and health related quality of life (HQRL) were measured at baseline and at 3 months. The primary outcome measure at 3 months was weight loss. Secondary outcomes included: body mass index (BMI), neck circumference (NC), waist circumference (WC), hip circumference $(\mathrm{HC})$ blood pressure $(\mathrm{BP})$, rectus femoris crosssectional area $\left(\mathrm{RF}_{\mathrm{CSA}}\right)$ and quadriceps maximal voluntary contraction (QMVC), 6 min walk distance (6MWD) and HRQL measures.

Results 37 subjects were randomised with data from 30 patients analysed at 3 months (15 in each group). There were no differences between the groups in all parameters measured at baseline. The intervention group showed greater weight loss than the control group $(-11.9 \pm 6.7$ vs. $-2.4 \pm 6.2 \mathrm{~kg} ; \mathrm{p}<0.0001)$. There were also differences in NC, WC and HC (all p $<0.001$, Table 1) with an improvement in BP observed in the intervention group (Table 1). In addition, there was an increase in weight corrected $\mathrm{RF}_{\mathrm{CSA}}$ and muscle strength $(\mathrm{p}<0.0001$, Table 1$)$ with an increase in $6 \mathrm{MWD}$ in the intervention group $(122 \pm 161$ vs. 46 $\pm 60 \mathrm{~m} ; \mathrm{p}=0.005$; Table1). Finally, HRQL improved in the intervention group as evidenced by a greater reduction in Epworth sleepiness score, an increase in severe respiratory insufficiency questionnaire sum score and a greater decrease in the hospital and anxiety depression score (Table 1 , all $\mathrm{p}<0.0001$ ).

Conclusion In patients with OHS, the addition of a hospitalhome hybrid personalised diet and exercise programme to standard NIV was shown to enhance weight loss as well as, skeletal muscle area and strength, exercise capacity and HRQL.

\section{REFERENCE}

1 Murphy PB, Davidson C, Hind MD, et al. Volume targeted versus pressure support non-invasive ventilation in patients with super obesity and chronic respiratory failure: a randomised controlled trial. Thorax. 2012;67:727-34

\section{REFERENCES}

1 Thorax 2010;65:829-32

2 Thorax 2012;67:1090-66 